\title{
LA RISCOPERTA DELLA VIA REGIA. FREUD LETTORE DI PLATONE*
}

\author{
Marco Solinas $^{* *}$
}

È soltanto alla fine, nella penultima pagina della prima edizione a stampa de L'interpretazione dei sogni, che Freud si richiama a Platone: ricordato l'episodio di un imperatore romano che fece giustiziare un suddito per un sogno di lesa maestà, scrive lapidariamente che «sarebbe ancora opportuno ricordare il detto di Platone: l'uomo virtuoso si limita a sognare quel che l'uomo malvagio fa nella vita» (Freud, 1899, p. 564) ${ }^{1}$. Questa citazione tardiva, estemporanea e come inaspettata, che riecheggia, depotenziandola, la trattazione del tiranno esposta nel IX libro de La Repubblica, racchiude una sfida ermeneutica che ritengo di rilevanza primaria ai fini sia della ricostruzione della genealogia teoretica, sia delle origini storiografiche della "scoperta" del sogno quale «via regia che porta alla conoscenza dell'inconscio» (Freud, 1899, p. 282).

Una sfida concernente la genealogia teoretica della via freudiana perché nei due brani cruciali del libro IX dai quali è desunto direttamente «il detto di Platone» non viene esposto semplicemente un aforisma edificante. Come mostrerò sinteticamente fin da subito, tralasciando qui l'analisi delle molteplici convergenze e divergenze tra le due teorie complessive (vedi Solinas, 2008), la concezione platonica poggia su una analisi rigorosa del sogno e su una correlata tematizzazione della follia che racchiude le due fondamentali intuizioni

\footnotetext{
* Questo articolo è un'ampia rielaborazione della nuova introduzione alla monografia in tedesco Via Platonica zum Unbewussten. Platon und Freud (Solinas, 2012), versione aggiornata e ampliata del libro Psiche: Platone e Freud (Solinas, 2008).

${ }^{* *}$ Università di Firenze, Dipartimento di Filosofia, Gruppo di ricerca in Storia della Filosofia. Recapito: Via dei Pecori 1, 50123 Firenze, E-Mail <marco.solinas.serra@gmail.com>.

${ }^{1}$ Il passo originale recita: «Wäre es noch am Platze, des Wortes Plato zu gedenken, dass der Tugendhafte sich begnügt, von dem zu träumen, was der Böse im Leben thut» (Freud, 1899, p. 370 della prima ediz. orig.).
}

Psicoterapia e Scienze Umane, 2012, XLVI, 4: 539-568

http://www.psicoterapiaescienzeumane.it 
poste alla base dell'intero edificio concettuale costruito ne L'interpretazione dei sogni. La prima è l'idea che la disamina dei sogni permetta di individuare la presenza e la natura di particolari desideri che sono stati oggetto di un processo di repressione tale da renderli solitamente inaccessibili allo sguardo della coscienza (paragrafo 1). La seconda è che la pressione di questi desideri repressi durante i normali stati di veglia, e in particolare di quelli di natura erotica, sia alla base di una molteplicità di stati psicopatologici (paragrafo 2). Posta l'originalità dell'edificio freudiano nella sua articolazione complessiva, resta fermo che le due intuizioni di fondo della «via regia» si sovrappongono a quelle di Platone.

La fugace citazione freudiana rappresenta nel contempo una sfida storiografica, sostanzialmente disattesa dalla critica ${ }^{2}$, ed è soprattutto a tale questione che è dedicato l'articolo, anzitutto perché vi sono delle prove testuali inequivocabili che mostrano che perlomeno fin dal periodo in cui compose L'interpretazione dei sogni Freud ebbe modo di leggere il primo lungo brano del libro IX, e due sue sintesi, nel quale è esposta la concezione del sogno quale manifestazione di desideri repressi. Poiché inoltre Freud aveva una conoscenza pregressa del pensiero platonico, e soprattutto dei fondamenti della psicologia elaborata ne La Repubblica, è più che verosimile ritenere che colse perlomeno la portata della prima cruciale intuizione del brano (paragrafo 3).

Acquisite queste prove mostrerò che è assai inverosimile ritenere che Freud abbia tralasciato di soffermarsi sulla concezione del libro IX, non cogliendone quindi la portata. Fin dagli anni 1880 egli nutrì infatti uno spiccato interesse per Platone, che rappresentò una significativa fonte di ispirazione, e che poi rincontrò ripetutamente negli anni seguenti, e ancora durante la stesura de L'interpretazione dei sogni, allorché continuò a coltivare lo studio del pensiero greco antico. Interessi, incontri e fonti tali per cui credo si possa sospettare che egli non soltanto abbia omesso più o meno deliberatamente di riconoscere la genealogia platonica della «via regia», ma abbia potuto trovare

\footnotetext{
${ }^{2}$ L'analogia tra le due concezioni oniriche venne sottolineata da Werner Jaeger fin dal 1947 (p. 64), in modo però del tutto accidentale e senza ipotizzare alcuna possibile influenza di Platone su Freud; tra i tanti lavori usciti nel corso degli anni sulle molteplici analogie tra la psicologia della Repubblica e Freud, nei quali però la questione del sogno è solitamente assente o del tutto secondaria, ricordo tra gli altri Kenny (1969), Simon (1973 pp. 91-122, 1978), Assoun (1976), Plass (1978, pp. 533-556), Gill (1985, pp. 1-26), Price (1990, 1995), Stella (1998); ha invece contemplato esplicitamente l'ipotesi di una influenza della Repubblica su Freud Sarah Kofman (1990), sebbene l'ipotesi venga solo schizzata e non venga apportata alcun indizio storiografico. Una rigorosa interpretazione in chiave freudiana della teoria della repressione ed emersione onirica esposta nei libri VIII e IX della Repubblica è stata offerta da Jonathan Lear (1998).
} 
ne La Repubblica una fonte tacita di ispirazione in una fase molto precoce, o persino ancor prima dell'inizio della composizione dell'opera (paragrafo 4).

Esposte le prove testuali sottostanti alla mia ipotesi, cercherò di render conto delle ragioni di fondo che potrebbero aver spinto Freud all'omissione, deliberata o invece frutto di una sorta di dimenticanza, riconducendole fondamentalmente al desiderio di mantenere il primato della "scoperta" della «via regia». Analizzerò poi l'ipotesi per cui Freud possa aver inserito il fugace richiamo al «detto di Platone» per neutralizzare la portata de La Repubblica, e in tal modo depistare la critica. Strategia che si sarebbe poi rivelata straordinariamente efficace, e che Freud avrebbe potuto adottare anche rispetto all'episodio correlato dell'imperatore romano e del sogno di lesa maestà, che vedremo ricalcare perfettamente quello del tiranno Dionisio di Siracusa, narrato da Plutarco e verosimilmente a lui noto (paragrafo 5).

Nel passo seguente sottolineerò che il sospetto di un utilizzo tacito delle intuizioni platoniche troverebbe conferma indiretta nel fatto che anche dopo l'uscita de L'interpretazione dei sogni Freud continuò ad attingere in modo piuttosto corposo dal lascito platonico, senza mai dichiarare la fonte dei temi rilanciati. Soltanto a distanza di diversi decenni, e in due casi specifici, si limitò ad esplicitare le convergenze con Platone presentandole come se fossero $a$ posteriori, mentre in altri due non riconobbe mai la fonte dei temi rilanciati, sebbene essa sia evidente (paragrafo 6).

Da ultimo proverò a tracciare un bilancio conclusivo delle ipotesi e dei dubbi via via analizzati.

\section{Via platonica e via regia}

Il detto di Platone citato da Freud (1899, p. 564), per cui i virtuosi si accontentano di sognare ciò che i malvagi fanno realmente, rimanda direttamente al brano con cui si apre il libro IX de La Repubblica. È il momento in cui, per spiegare le dinamiche intrapsichiche e sociopolitiche che conducono alla formazione del tiranno (l'ultimo stadio della fenomenologia degenerativa presentata nel libro VIII), Platone si sofferma sulla particolare specie di desideri che caratterizza tale figura. Si tratta dei desideri definiti «fuorilegge» o «contrari alle leggi» (paranomoi: La Repubblica, 571b4), facenti parte di quelli non necessari (distinti dai bisogni primari-necessari quali fame e sete), soggetti usualmente a particolari forme di repressione da parte dell'istanza razionale (e morale). Repressione tale per cui, pur essendo presenti e attivi nel quadro delle dinamiche intrapsichiche, essi sfuggono usualmente allo sguardo della coscienza, manifestando la loro presenza in sogno: 
«Proprio lui ci resta da esaminare, dissi io, l'uomo tirannico: come si trasforma a partire da quello democratico, e, una volta formatosi, che tipo di uomo è e qual è il suo modo di vita, sventurato o felice. - Ci resta appunto questo, disse - Sai allora, dissi io, che cosa desidero ora? - Che cosa? - Discutere la questione dei desideri: quali e quanti siano, non mi sembra che l'abbiamo ancora distinto in modo adeguato. Ma se questo resta incompiuto, l'indagine che vogliamo condurre sarà meno chiara. - Ma non siamo ancora in tempo?, disse lui - Certo. E guarda che cosa voglio scorgere in essi. È questo: fra i piaceri e i desideri non necessari, alcuni mi sembrano essere contrari alle leggi. Essi probabilmente nascono in ognuno, ma se vengono repressi (kolazomenai) dalle leggi e dai desideri migliori insieme con la ragione, nel caso di alcuni uomini si allontanano del tutto oppure restano pochi e deboli, in altri più forti e numerosi. - Quali sono, disse, questi desideri di cui parli? - Quelli, dissi io, che si risvegliano nel sonno, quando il resto dell'anima, ciò che vi è in essa di razionale, socievole e adatto al comando riposa, mentre la parte ferina e selvaggia, piena di cibo o di vino, si sfrena nella sua danza, e, scacciando il sonno, cerca di aprirsi la via per dare sfogo ai suoi abituali costumi. Sai bene che in un simile stato osa fare di tutto, come sciolta e sbarazzata da ogni freno di vergogna e ragionevolezza. Tentare di accoppiarsi con la madre (così pensa), non la imbarazza affatto, o con chiunque altro fra uomini, dèi, animali, e commettere qualsiasi assassinio, e non astenersi da alcun cibo: in una parola non si arresta di fronte agli estremi della follia e della spudoratezza. - Dici cose verissime, rispose» (Platone, La Repubblica, 571a-d).

Platone individua dunque nel sogno il canale privilegiato che permette di accertare la presenza e di chiarire la natura di particolari desideri che, nel quadro delle opposizioni e dei conflitti tra le diverse istanze intrapsichiche, sono stati letteralmente oggetto di una "repressione" (nei termini classici freudiani di una "rimozione”)", che ne impedisce la manifestazione negli stati di veglia. Modalità repressiva peraltro già richiamata nel libro VIII in relazione all'analisi delle figure dell'oligarchico e del democratico (che precedono quella del tiranno), e che risultava lasciare aperta la possibilità ai desideri repressi di tipo non necessario di prolificare e di rinvigorirsi segretamente, «nell'ombra», finendo così «per conquistare l'acropoli dell'anima del giovane, accorgendosi che è vuota di conoscenze, di belle occupazioni e di discorsi veri, che sono i migliori guardiani e difensori nella mente di uomini cari agli dèi» ( $\mathrm{La}$ Repubblica, 560a-c). Restando al brano di apertura del libro IX, emerge in breve che i desideri fuorilegge, letteralmente repressi, approfittano del cedimento dell'attività di controllo dell'istanza razionale che avviene durante il sonno: si manifestano in sogno e mostrano così la loro natura più terrificante $\mathrm{e}$ inconfessabile, ivi incluso quel desiderio che Freud definirebbe senza indugio come "edipico".

\footnotetext{
${ }^{3}$ Sulle diverse strategie di repressione, allontanamento e trasformazione dei desideri approntate da Platone nella Repubblica e confrontate alle modalità e ai meccanismi delineati da Freud, mi permetto di rimandare a Solinas (2008, capitoli 2-3).
} 
Il brano viene quindi a offrire una limpida testimonianza della convergenza con la posizione rilanciata da Freud ne L'interpretazione dei sogni: il sogno, inteso quale soddisfacimento di desiderio, rappresenta un canale privilegiato per accertare la presenza e mostrare la natura di quei desideri che vengono usualmente rimossi - posto che in questo testo la rimozione (Verdrängung) è presentata come analoga alla repressione (Unterdrücken) ${ }^{4}-\mathrm{e}$ che durante il sonno approfittano dell'indebolimento o della totale sospensione dell'attività di controllo delle istanze censorie. Nocciolo teorico così sintetizzato nella prima edizione de L'interpretazione dei sogni:

«Volendoci accontentare di un minimo accrescimento di conoscenza assolutamente certa, diremo: il sogno ci dimostra che il materiale represso [das Unterdrückte] continua a sussistere anche nell'uomo normale e rimane capace di prestazioni psichiche. Il sogno stesso è una delle manifestazioni di questo materiale represso; secondo la teoria lo è in tutti i casi, secondo l'esperienza concreta almeno in un gran numero di casi, nei quali saltano agli occhi nel modo più chiaro i sorprendenti caratteri del sogno. Ciò che è represso psichicamente, ciò che nella vita vigile è stato ostacolato nella propria espressione dalla reciproca eliminazione delle contraddizioni, ed escluso dalla percezione interna, trova nella vita notturna, e sotto il dominio delle formazioni di compromesso, mezzi e vie per imporsi alla coscienza.

Flectere si nequeo Superos, Acheronta movebo» (Freud, 1899, p. 553, p. 362 prima ediz. orig. tedesca, corsivi nell'originale).

Brano a cui nella seconda edizione, del 1909, Freud farà seguire una definizione destinata a divenire celebre: «Ma l'interpretazione del sogno è la via regia che porta alla conoscenza dell'inconscio nella vita psichica» (ibid., p. 553 , corsivi nell'originale).

Certo nel testo freudiano tutti i sogni vengono interpretati quali soddisfacimenti di desideri, sì che viene a dischiudersi un'opera interpretativa, di decodificazione e smascheramento, che permette l'analisi dei meccanismi propri

\footnotetext{
${ }^{4}$ Vedi Freud (1899): «(...) si trovano nella vita psichica desideri rimossi (...). "Si trovano" non è inteso storicamente, nel senso che simili desideri sono esistiti e poi sono stati distrutti; per la teoria della rimozione - cui bisogna ricorrere nelle psiconevrosi - simili desideri rimossi esistono ancora, ma contemporaneamente esiste un'inibizione che pesa su di essi. Il linguaggio colpisce nel giusto quando parla della repressione [Unterdrücken] di tali impulsi. L'organizzazione psichica, che permette a codesti desideri repressi di realizzarsi, rimane intatta e utilizzabile» (p. 220, corsivi nell'originale); in questo testo Freud si esime esplicitamente dall'operare una chiara distinzione tra repressione e rimozione; vedi anche Freud, 1899: «(...) ho evitato di indicare se alla parola "represso" [unterdrückt] io dia un significato diverso che alla parola "rimosso" [verdrängt]. Potrebbe essere risultato chiaro solo il fatto che quest'ultima accentua più vigorosamente della prima l'appartenenza all'inconscio» (p. 552, nota 1). Nel testo Freud continuerà infatti a parlare di "represso" intendendolo quale sinonimo di "rimosso".
} 
del sistema inconscio e delle diverse forme in cui opera l'attività censoria delle istanze morali, assente ne La Repubblica. Per Platone, infatti, o i desideri repressi emergono limpidamente, oppure la parte razionale resta desta durante il sonno, impedendo la loro manifestazione e permettendo, in positivo, la genesi di una seconda categoria di sogni. Categoria alla quale è attribuito un particolare valore di verità, anche di natura apparentemente premonitrice, come viene spiegato dopo la descrizione del sogno "edipico":

«Quando invece, credo, un uomo si trovi in una condizione di sanità e moderazione, e si accosti al sonno dopo aver tenuto ben desta la sua parte razionale, nutrendola con buoni discorsi e ricerche, ed essersi concentrato nella riflessione, senza d'altra parte aver affamata né troppo riempita la parte desiderante, in modo che essa si assopisca e non turbi quella migliore con lo strepito della sua soddisfazione o dei suoi lamenti, e permetta invece che quest'altra - sola in se stessa nella sua purezza - indaghi e cerchi di percepire ciò che non conosce, che si tratti di qualcosa che appartiene al passato, al presente $o$ al futuro; e similmente avendo ammansita anche la parte aggressiva, per non addormentarsi con la collera in subbuglio per essersi adirata contro qualcuno, bensì, calmati entrambi questi aspetti dell'anima e attivato invece il terzo, nel quale risiede il pensiero, si conceda in questo stato di riposo - ti renderai conto che soprattutto in esso può venire in contatto con la verità e che allora le visioni fantasticate nei sogni sono le meno contrarie alle leggi» (Platone, La Repubblica, 571d-572a).

Poste queste due differenti categorie di visioni oniriche, resta fermo che nel libro IX viene inaugurato un approccio in virtù del quale una prima fondamentale categoria di sogni rientra pienamente ed esclusivamente nell'ambito dei fenomeni psicologici naturali. La loro formazione dipende dai rapporti di forza tra le tre istanze in cui Platone divide il complesso psichico: quando la parte desiderante e quella aggressiva restano deste, i sogni debbono essere intesi quale diretta espressione di quei desideri che pur essendo stati repressi continuano a permanere in esse. E Platone, come farà poi Freud, conclude questa prima parte dell'analisi sottolineando che si tratta di una dinamica universale, concernente anche quegli uomini «virtuosi» richiamati nel motto citato ne L'interpretazione dei sogni:

«Però parlando di queste cose siamo andati troppo lontani. Ma ciò che vogliamo capire è questo: in ognuno - anche in quei pochi di noi che sembrano essere del tutto moderati - è senza dubbio presente una forma di desideri terribile, selvaggia e illegale, che si manifesta chiaramente appunto nel sonno» (Platone, La Repubblica, 572b).

La via regia freudiana si sovrappone alla via platonica. 


\section{Sogno e follia}

Il detto platonico citato da Freud diviene poi perfettamente chiaro in entrambi i sensi allorché si consideri il brano del libro IX nel quale Platone spiega che nel tiranno, il malvagio per eccellenza, i desideri repressi possono svincolarsi dalle catene che ne relegano la manifestazione al sogno, imponendo la loro soddisfazione reale:

«(...) quelle vecchie opinioni che possedeva fin da bambino su ciò che è bello e ciò che è vergognoso - ed era ben convinto che fossero giuste - verranno ora sopraffatte da nuove convinzioni, da poco affrancate dalla schiavitù, che fanno da scorta a Eros e che da lui sono aiutate: quelle che prima, quando egli era ancora sottomesso alle leggi e al padre, e si autogovernava in modo democratico, si liberavano solo in sogno, nel sonno. Ma sotto la tirannide di Eros, divenuto in ogni momento della sua vita da desto quello che raramente gli capitava di essere in sogno, non si asterrà da alcun tremendo assassinio né da alcun cibo né da alcuna azione: Eros, che vive in lui da tiranno in piena anarchia e illegalità, in quanto è egli stesso un monarca, condurrà chi lo ospita in sé come in una città a ogni forma di audacia, onde nutrire se stesso e la turba che lo circonda (...)» (Platone, La Repubblica, 574d-575a).

Più da vicino, qui Platone sta spiegando, sul doppio piano psicologico e sociale, il passaggio che dal carattere democratico conduce alla formazione del tiranno. Nel primo caso, i desideri e le opinioni che accompagnano Eros, seppur ormai numerosi e vigorosi, si trovano in uno stato di «schiavitù» tale per cui la loro soddisfazione e invero la loro stessa manifestazione è limitata al sogno. Viceversa, nel tiranno, che rappresenta il grado estremo di degradazione, Eros dilaga, giungendo a esercitare una vera e propria tirannia intrapsichica, sì da imporre la soddisfazione reale dei desideri più tremendi, ora liberati dalla schiavitù. È questo il senso della definizione secondo cui il tiranno è «divenuto in ogni momento della sua vita da desto quello che raramente gli capitava di essere in sogno» (ibid.). Ed è questa la definizione che rende pienamente conto del motto richiamato da Freud (1899, p. 564) per cui "i virtuosi si limitano a sognare ciò che i malvagi fanno realmente”.

Per Platone tale analisi non mette però soltanto in luce il fatto che ogni uomo, quindi anche chi è virtuoso, alberga in sé dei desideri "immorali" che si palesano nel sogno - idea peraltro espressa da diversi autori ottocenteschi puntualmente citati ne L'interpretazione dei sogni - e che il malvagio attua nella realtà. Allorché l'acropoli intrapsichica è occupata da Eros, che impone senza mediazioni di sorta la sua tirannia assoluta, il tiranno viene difatti nel contempo a ritrovarsi in una condizione che Platone descrive come letteralmente psicopatologica. Come è chiarito nelle circa quattro o cinque pagine 
che dal brano di apertura sul sogno giungono a quello sulla tirannia di Eros, l'uomo tirannico in senso stretto deve difatti essere considerato come colui che è in una condizione strettamente e gravemente patologica: «Un uomo diviene tirannico in senso stretto, mio divino amico, quando o per natura, o per modo di vita, o per entrambi, sia diventato al tempo stesso ubriacone, erotico e melancolico» (La Repubblica, 573c). Punto fondamentale ribadito ripetutamente anche nel seguito del libro IX: «E un'anima soggetta a tirannide vedrà ridotta al minimo la possibilità di agire secondo la propria volontà, s'intende dal punto di vista dell'anima nel suo insieme: anzi, sempre trascinata dalla violenza di quel furore, sarà piena di turbamento e di instabilità» (ibid., 577d); e ancora: «Ritieni che simili patimenti siano presenti in qualcun altro più che in quest'uomo tirannico reso folle dai suoi desideri erotici?» (ibid., 578a).

L'analisi del sogno permette quindi di spiegare il processo di formazione degli stati deliranti del tiranno anzitutto perché consente di individuare la forma e la natura di quei desideri repressi e tenuti in schiavitù che, guidati da Eros, hanno infine conquistato il potere assoluto nel quadro dei conflitti e dei rapporti di forza tra le diverse istanze intrapsichiche. L'intera analisi inaugurata dalla disamina dei desideri non necessari che emergono nel sogno mira del resto a questo obiettivo: mostrare che il tiranno è nel contempo il più malvagio, infelice e malato degli uomini. Al di là del taglio etico, morale e politico, sul piano psicologico tale concezione racchiude quindi anche la seconda intuizione posta alla base della tematizzazione freudiana delle correlazioni tra sogno e stati psicopatologici. L'elemento cardinale che differenzia l'approccio adottato ne L'interpretazione dei sogni dalle classiche analogie tra sogno e follia tracciate nel corso dell'intera storia del pensiero occidentale, infatti, è che per Freud sia nei sogni sia negli stati nevrotici si ha essenzialmente a che fare con la presenza e la pressione di desideri repressi nel quadro dei conflitti tra istanze contrapposte. Desideri repressi che approfittano del cedimento delle istanze di controllo per emergere nel sogno; e desideri repressi la cui pressione e conquista del potere intrapsichico nella veglia determina differenti tipi di stati psicopatologici. In breve, sia in Platone sia in Freud il sogno rappresenta il canale privilegiato per individuare la presenza di quei desideri, soprattutto di natura erotica, oggetto di particolari modalità repressive, la cui attività è alla base della formazione e dello sviluppo di una molteplicità di stati patologici più o meno gravi. Anche da questa prospettiva, la via regia freudiana si sovrappone alla via platonica.

Certo ne L'interpretazione dei sogni l'analisi delle differenti forme di correlazione tra sogno, nevrosi e psicosi è sviluppata in modo incomparabilmente più articolato, ricco e complesso che non nei brevi passi de La Repubblica, elaborati peraltro costantemente sul doppio piano psicologico ed etico- 
politico. Ciò nondimeno, l'analogia tra le due intuizioni di fondo è pregnante e inequivocabile. Inoltre, lo stesso Freud pratica spesso una spiccata politicizzazione della sfera psicologica che lo avvicina ancor di più alla prospettiva platonica, come emerge ad esempio ove spiega il rapporto tra sogno e psicosi ricorrendo all’immagine di una «fortezza» (Festung) psichica, sovrapponibile al modello platonico dell'acropoli:

«I moti di desiderio inconsci tendono evidentemente a imporsi anche di giorno e sia il fenomeno della traslazione sia le psicosi ci dimostrano che essi vorrebbero farsi strada, passando per il sistema del preconscio, sino alla coscienza e al dominio della motilità. Nella censura tra Inc [inconscio] e Prec [preconscio], che il sogno addirittura ci costringe ad ammettere, dobbiamo dunque riconoscere e rispettare il guardiano della nostra salute mentale. Ma in questo caso, non è forse imprudente, da parte del guardiano, diminuire nottetempo la sua attività, permettere che si esprimano i moti repressi dell'Inc, rendere nuovamente possibile la regressione allucinatoria? Penso di no, perché quando l'attento guardiano va a riposare - abbiamo però le prove che non dorma profondamente - chiude anche la porta che conduce alla motilità. Quali che siano gli impulsi dell'Inc, normalmente inibito, che si agitano sulla scienza, possiamo concedere loro ampia libertà; essi rimangono innocui, perché non sono in grado di azionare l'apparato motorio, l'unico che possa influenzare, mutandolo, il mondo esterno. Lo stato di sonno garantisce la sicurezza della fortezza da sorvegliare. La situazione è meno tranquilla quando lo spostamento delle forze è prodotto non dalla riduzione notturna dell'impiego di energie della censura critica, ma da un loro indebolimento patologico, o da un rafforzamento patologico degli eccitamenti inconsci, mentre il preconscio è investito e le porte della motilità sono aperte. Allora il guardiano viene sopraffatto, gli eccitamenti inconsci sottomettono il preconscio, dominano da esso le nostre parole e le nostre azioni, oppure conquistano con violenza la regressione allucinatoria e dirigono l'apparato (non a essi destinato) in virtù dell'attrazione che le percezioni esercitano sulla ripartizione della nostra energia psichica. È questo lo stato che chiamiamo psicosi» (Freud, 1899, p. 517).

\section{Gli incontri con il libro IX}

Freud utilizzò in modo corposo, citandola ripetutamente fin dalla prima edizione de L'interpretazione dei sogni, la dissertazione di Paul Wilhelm Radestock, allievo di Wilhelm Wundt, intitolata Schlaf und Traum ("Sonno e sogno”), edita a Lipsia nel 1879, e presto recepita a livello internazionale. In questo testo Radestock, che oltre a La Repubblica rimanda spesso a molti altri dialoghi platonici, mentre discute del rapporto tra stimoli e sogni erotici e morali, in una lunga nota scrive: 
«Qui è confermato il vecchio detto per cui i buoni si concedono soltanto in sogno ciò che i malvagi fanno da svegli. È come se la natura volesse compensare, incantando lo spirito con ricche immagini, ciò che la coscienza morale e il duro lavoro della veglia proibiscono» (Radestock, 1879, p. 297, nota 190).

Precisamente il detto che Freud riprenderà e riporterà in termini quasi identici nel suo testo. Richiamato il detto, Radestock cita poi i tre versi sul sogno incestuoso dell'Edipo re e, rimandando a «Plato republ. IX, p. 571», riporta le frasi (direttamente in greco, che Freud conosceva bene) in cui leggiamo: «Tentare di accoppiarsi con la madre (così pensa), non la imbarazza affatto, o con chiunque altro fra uomini, dèi, animali, e commettere qualsiasi assassinio, e non astenersi da alcun cibo».

Radestock insiste poi sulla dimensione morale: «Platone certo dice che soltanto nelle nature sensibili le brame selvagge regnano sfrenatamente, però anche gli antichi padri della Chiesa si sono ripetutamente lamentati dell'immoralità dei loro sogni» (ibid.). Dello stesso tenore un'altra considerazione, esposta nel corpo del testo, per cui Hildebrandt avrebbe seguito «l'opinione di Platone», secondo la quale «un uomo perfetto non potrebbe mai sognare qualcosa di impuro. Più la vita è pura, più lo sarebbero i sogni; più si è impuri, più impuri sono i sogni» (p. 163) ${ }^{6}$. Infine, in altre due occasioni Radestock (1879, pp. 39, 186 e note), rimandando a La Repubblica, IX 571d-572a, sottolinea che Platone intende i sogni anche come momenti in cui è possibile giungere a una conoscenza di tipo premonitrice.

In secondo luogo, nel breve testo di Bernhard Büchsenschütz Traum und Traumdeutung im Alterthume ("Sogno e interpretazione dei sogni nell'antichità”), anch'esso citato e utilizzato fin dalla prima edizione de L'interpretazione dei sogni, e dedicato principalmente alla questione dei sogni profetici e all'arte divinatoria nell'antichità, l'autore offre la seguente sintesi della concezione platonica:

«Platone, sebbene abbia trattato la questione soltanto accidentalmente, ha analizzato la natura dei sogni in accordo alla sua psicologia, mirando principalmente a mostrare che la diversità dei sogni deve essere ricondotta alle diverse condotte delle tre parti dell'anima. Quando infatti - dice Platone [rimando alla nota 37: La Repubblica, IX 571c] - la parte razionale e pensante dell'anima [to logistikon] dorme, quella animale

\footnotetext{
${ }^{5}$ Nell'originale: «Hier bewahrheitet sich das alte Wort, dass die Guten sich nur im Traume erlauben, was die Schlechten im Wachen thun»; nei suoi brevi ma frequenti rimandi a Platone, Radestock si richiama soprattutto a Fedro, Apologia, Timeo, Filebo e Fedone.

${ }^{6}$ Qui Radestock cita letteralmente due considerazioni sulla responsabilità ed eticità dei sogni esposte da F.W. Hildebrandt (1875), alle pagine 53 e 54, reinterpretandole autonomamente, poiché nella discussione della questione Hildebrandt di fatto non si richiama a Platone.
} 
[to theriodes te kai agrion] invece, ripiena di cibi e bevande, agitandosi con vigore, allontana da sé il sonno e cerca di realizzare le sue attitudini, è allora che sorgono i sogni terrificanti, irrazionali e impudichi. Se invece la persona tiene desta la parte razionale dell'anima, dopo averla nutrita con bei discorsi e riflessioni, e non lascia che la parte desiderante si agiti a causa di eccesso o di mancanza di cibo, e allo stesso modo calma la terza parte dell'anima, quella coraggiosa [to thymoeides], allora egli, una volta addormentatosi, raggiunge in tale condizione la più alta verità. Ne consegue che i sogni di ogni tipo non sono altro che delle attività autonome dell'anima che seguono le stesse leggi valide durante la veglia, sì che, anche in sogno, tanto più l'anima si occupa della conoscenza del vero, tanto più l'attività dei suoi elementi fisici è respinta indietro e, dunque, il raggiungimento di questa conoscenza non necessita di un particolare influsso da parte della divinità. Di una forza profetica dei sogni non viene dunque fatto cenno, anche se, poiché la verità è eterna, alla sua conoscenza è correlata la conoscenza del futuro» (Büchsenschütz, pp. 16-17 e note a p. 77; vedi la nota 38 in: La Repubblica, IX, 572a).

In terzo luogo, l'intero brano saliente sul sogno del libro IX, dalla premessa in cui viene spiegato che si tratta di desideri repressi fino alle modalità suggerite da Platone per prevenirne l'emersione onirica, è fedelmente riportato da Cicerone nel primo libro del De Divinatione (XXIX, 60-61). Testo classicissimo che Freud cita esplicitamente fin dalle prime pagine della prima edizione de L'interpretazione dei sogni. Dunque, Freud ebbe modo di leggere il brano de La Repubblica per intero, e probabilmente lo lesse effettivamente.

Oltre a queste tre fonti, Freud riportò in bibliografia anche il testo di Emil Richard Pfaff, Das Traumleben und seine Deutung nach den Principien der Araber, Perser, Griechen, Inder und Aegyptier ("La vita onirica e la sua interpretazione secondo i princìpi degli arabi, persiani, greci, indiani ed egiziani”), edito nel 1868, nel quale vi è una sintesi della concezione platonica del sogno (pp. 15-16, 109). Poiché però l'unica citazione di un suo brano venne fatta indirettamente attraverso il lavoro di Heinrich Spitta, si può dubitare che Freud lo lesse con attenzione ${ }^{7}$. L'aforisma è altresì riportato da Max Leidesdorf (1880) nella discussione sulla responsabilità dei sogni nel suo lavoro Das Traumleben, anch'esso citato in bibliografia, ove è però definito genericamente come «un antico detto popolare» (p. 13) ${ }^{8}$.

Riepilogando, in un periodo che al più tardi è sincronico alla stesura de L'interpretazione dei sogni, Freud probabilmente lesse il primo lungo brano del libro IX, e di certo lesse la parte sul sogno edipico, un suo commento e

\footnotetext{
${ }^{7}$ Citato da Freud in bibliografia, e di cui viene riportato un brano, a pagina 36 della prima edizione, attraverso Spitta (1882).

${ }^{8}$ In originale: «Im Traume fehlt uns jede sittliche Beurtheilung und Werthschätzung, man begeht die unangemessensten Dinge mit der größten Unbefangenheit. „Der gute Mensch erlaubt sich nur im Träume, was der Böse im Wachen thut“ ist ein altes Volksdiktum».
} 
una sintesi. Tali letture vennero inoltre coadiuvate da una conoscenza pregressa de La Repubblica, con cui Freud aveva di certo avuto modo di familiarizzarsi perlomeno fin dal 1879, allorché l'antichista viennese Theodor Gomperz, su indicazione di Franz Brentano, gli affidò la traduzione di un lungo articolo di John Stuart Mill (1866), di circa 70 pagine, edito in tedesco nel $1880^{9}$, interamente dedicato all'opera di George Grote su Platone. Articolo che rappresenta una sorta di panoramica critica del pensiero soprattutto etico e morale platonico, che dedica molto spazio a La Repubblica, di cui vi è una lunga citazione letterale (tratta dal libro VI) che verosimilmente condusse il giovane Freud a visionare il testo di prima persona.

Tra le altre cose, nell'articolo Mill richiama peraltro anche la figura del tiranno tracciata nel libro IX, seppur non in relazione al sogno:

«Nella combinazione di profondità filosofica, eccelsa eloquenza e ricca immaginazione poetica che dà corpo agli ultimi libri de La Repubblica vi è una pregnante raffigurazione dei modi in cui la società, con le sue tentazioni, i suoi elogi e le sue condanne mal riposti corrompe questi caratteri originariamente elevati: il ritratto del tiranno devastato, la consumazione della sua colpa, il suo dispregio di dèi e uomini, l'abisso della sua miseria interiore, e il castigo che lo attende, già in questa vita e di certo nel mondo che verrà, è uno dei passaggi di Platone più conosciuti e più impressionanti» (Mill, 1880 pp. 106-107, p. 361 ediz. inglese).

Freud si fece dunque certamente una prima idea della trattazione del tiranno del libro IX fin dal momento della traduzione - lavoro che com'è noto richiede particolare attenzione nei confronti dei testi tradotti, e che come detto necessitò verosimilmente di una consultazione diretta del grande dialogo - ed ebbe altresì modo di conoscere i fondamenti della tripartizione psichica platonica, la medesima che emerge nell'analisi dell'emersione onirica, di cui tradusse la seguente sintesi:

«Ne La Repubblica (...) la giustizia equivale alla piena supremazia della ragione nell'anima. L'anima umana è divisa nei celebri tre elementi: razionale, spirito o passionale [to thymoeides, un altro modo misto], e concupiscibile. L'anima giusta è quella in cui ognuno dei tre elementi svolge il ruolo che gli spetta; nella quale cioè la ragione domina, l'elemento passionale è di ausilio alla ragione, ed entrambi uniti mantengono la concupiscibile in una condizione di obbediente sottomissione» (Mill, 1880 p. 83, p. 341 ediz. inglese).

\footnotetext{
${ }^{9}$ Freud lavorò alla traduzione di Mill soprattutto nell'agosto del 1879, vedi la lettera a Silberstein del 10 agosto 1879: «En fin que tengo tiempo y ocio para escribirte habiendo trabajado las dos semanas anteriores á la traduccion de tal ensayo por J.S. Mill sebre Plato y sus obras» (Freud, 1871-81, p. 138).
} 
Tripartizione psichica che Freud peraltro molto probabilmente aveva incontrato ancor prima, ancora una volta grazie a Brentano, di cui seguì assiduamente i corsi universitari di filosofia (negli anni 1873-76), e per il quale nutrì grandissima stima (vedi Schellenbacher, 2011; Freud, 1991, p. 55). Nel suo capolavoro, La psicologia dal punto di vista empirico, edito nel 1874, Brentano si richiamava difatti ripetutamente a La Repubblica, e nel breve sottocapitolo intitolato La distinzione di Platone tra parte concupiscibile, irascibile e intellettiva dell'anima, sintetizzava l'isomorfismo psiche-polis e sottolineava la centralità conferita da Platone ai conflitti intrapsichici in relazione alle pulsioni e alle diverse parti dell'anima, considerandolo come il primo autore ad aver dischiuso la via all'analisi della divisione psichica ${ }^{10}$.

\section{Interessi e ispirazioni}

Nonostante questi incontri reiterati si potrebbe però ipotizzare che Freud, seppur ricordò e citò il motto platonico, lesse frettolosamente e con disattenzione le fonti sopra riportate. Fondamentalmente disinteressato alla concezione dell'antico filosofo, potrebbe insomma non avere apprezzato né colto pienamente la portata del brano, e per questa ragione essersi limitato a un richiamo fugace: poco più che uno sfoggio di erudizione. Vi sono però degli indizi piuttosto stringenti che rendono tale ipotesi assai inverosimile, e inducono nel contempo a ritenere che Freud abbia invece assimilato e poi in certo qual modo dimenticato la teoria platonica, oppure abbia omesso deliberatamente di riconoscere la genealogia platonica della via regia, utilizzando il libro IX quale fonte tacita di ispirazione.

L'ipotesi del disinteresse stride anzitutto con il fatto che Freud rimase fortemente impressionato dalla teoria della reminiscenza platonica fin da quando tradusse il saggio di Mill. Fu lui stesso a confidarlo a Ernest Jones (1953-57), che riportò l'affermazione nel seguente brano della sua monumentale biografia; un breve brano volto (paradossalmente) a sminuire l'influenza platonica, che è poi divenuto un riferimento canonico per la ricostruzione dei rapporti con Platone:

\footnotetext{
${ }^{10}$ Brentano tra le altre cose dice: «Il merito di aver aperto la strada spetta a Platone. Egli distinse tre classi fondamentali di fenomeni psichici o piuttosto, come egli si espresse, tre parti dell'anima, ognuna delle quali comprendeva particolari attività psichiche, vale a dire la parte concupiscibile [to epithymetikon], irascibile [to thymoeides] e razionale [to logistikon]. (...) Egli trovò nell'uomo una lotta tra opposti, prima tra le esigenze della ragione e degli istinti sensibili e poi anche tra gli stessi istinti sensibili» (Brentano, 1874, vol. 2, pp. 22-23; edizione originale del 1874: Inhaltsverzeichnis, p. XII: «Fünftes Kapitel, § 1. Platon’s Unterscheidung eines begierlichen, zornmüthigen und vernünftigen Seelentheiles», sviluppato alle pp. 233-236).
} 
«Il quarto [saggio], dello stesso Mill, era sul Platone di Grote. Molti anni dopo (nel 1932) Freud notò che la sua conoscenza della filosofia platonica era molto frammentaria, e ciò significa forse che tutto ciò che ne sapeva l'aveva appreso appunto dal saggio di Mill. Egli aggiungeva però che la teoria di Platone sulla reminiscenza, che Mill trattava assai bene, l'aveva molto colpito, e che in un certo periodo gli aveva dato molto da pensare. Molto tempo dopo, infatti [nel 1920], egli introdusse alcuni suggerimenti di Platone nel suo libro Al di là del principio di piacere» (Jones, 1953-57, vol. 1, p. 87).

Va da sé che la deduzione personale di Jones è fallace, poiché Freud aveva studiato Platone già al liceo, approfondendo in particolare l'Apologia di Socrate e il Critone, e dunque l'antico filosofo era un autore a lui ben noto fin da giovanissimo ${ }^{11}$. Se poi Freud dichiarò che ebbe una conoscenza frammentaria della filosofia platonica, ciò naturalmente non significa che non avesse letto il libro IX de La Repubblica. Venendo alla teoria della reminiscenza, alla quale Mill (1880 pp. 64 sg., pp. 326 sg. ediz. inglese) dedicava effettivamente particolare attenzione, possiamo presumere che essa in un modo o nell'altro contribuì alla formulazione della teoria dell'isteria, di cui uno dei pilastri è rappresentato dalla tesi per cui «l'isterico soffrirebbe per lo più di reminiscenze»

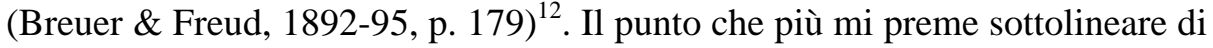
questa testimonianza è però che qui emerge in modo cristallino il fatto che Freud trovò nel pensiero di Platone un importante spunto di riflessione, che poi verosimilmente utilizzò, tacitamente, nei primi anni 1890.

Sempre gli Studi sull'isteria mostrano peraltro che Freud e Breuer trassero ispirazione, ancora una volta tacita, anche da un'altra fonte antica: la teoria della catarsi di Aristotele, che era certamente nota ai due autori per via dell'interpretazione offerta dall'antichista Jacob Bernays (zio della moglie di Freud, Martha Bernays) e da Gomperz (vedi Hirschmüller, 1978, pp. 206 sg.; Vöhler \& Linck, 2009). E ancora, nonostante nell'Autobiografia Freud (1924) scrisse poi che fu attraverso un articolo di Havelock Ellis del 1898 che venne a conoscenza del legame tra isteria e sessualità esplicitato nel Timeo ${ }^{13}$, si può

\footnotetext{
${ }^{11}$ Vedi Hemecker (1991, p. 40 e nota 140), che rimanda al Neunter Jahresbericht des Leopoldstädter Communal-Real- und Obergymnasiums in Wien, 1873, Schularchiv des RainerGymnasium in Wien.

12 Molto più tardi Freud (1915-17) sottolineerà peraltro anche la vicinanza tra la teoria dell'isteria e le «note dottrine socratiche, secondo le quali persino i vizi si basano sull'ignoranza» (p. 442).

${ }^{13}$ Vedi Freud (1924): «Inoltre, a quell'epoca, non sapevo neppure che facendo risalire l'isteria alla sessualità mi ero riportato alle epoche più antiche della medicina e addirittura alle concezioni di Platone. Appresi queste cose solo più tardi, da un saggio di Havelock Ellis» (p. 92; vedi Ellis, 1898). Sulla lettura dell'articolo vedi anche Lettera a Fliess del 3 gennaio 1899: «Una cosa piacevole, della quale intendevo scriverti già ieri, viene da Gibilterra, dal signor Ha-
} 
nutrire il sospetto che avesse letto il brano platonico prima di iniziare gli Studi sull'isteria: nel Traité clinique et thérapeutique de l'hystérie di Gilles de la Tourette, il cui primo volume fu pubblicato nel 1891 con una prefazione di Charcot, il passo è difatti fedelmente riportato fin dalla seconda pagina:

«La storia dell'isteria comincia, dal punto di vista documentario, con i più antichi scritti medici e filosofici, poiché nell'antica Grecia, culla di tutte le scienze, la filosofia era assai prossima alla medicina, quando non l'inglobava nel suo dominio. E così, lungo questa via, Democrito e Platone furono senza alcun dubbio i precursori della medicina; e non fu forse Platone il primo a formulare la teoria che per lungo tempo guidò la clinica e la terapia della nevrosi, allorché scrisse: "L'utero è un animale che desidera ardentemente generare dei bambini. Se resta per molto tempo sterile dopo la pubertà, lo sopporta male, s'indigna, ed erra per tutto il corpo, ostruendo le uscite dell'aria, impedendo la respirazione, conducendo il corpo a dei gravi pericoli e talvolta a svariate malattie, fintanto che il desiderio e l'amore, congiungendo l'uomo e la donna, fanno nascere un frutto, e lo colgono come da un albero [nota: Euvres de Platon. Trad. di Corsin. Tomo XII, p. 242]”. Questa citazione del Timeo, che riportiamo dopo tanti altri, racchiude il famoso aforisma "Nubat illa et morbus effugiet" [la si mariti e il morbo sparirà], che per sì lungo tempo sarà alla base della teoria e della terapia dell'isteria femminile (...)» (Tourette, 1891, pp. 1 sg.).

A ogni modo, resta fermo che perlomeno fin dai primi del 1899 Platone si dovette configurare agli occhi di Freud come un autore particolarmente interessante anche dalla prospettiva del legame tra isteria e sessualità. E sempre in questo periodo, mentre lavorava febbrilmente al completamento de L'interpretazione dei sogni (consegnata all'editore nel settembre del 1899), Freud continuò peraltro a coltivare piuttosto intensamente lo studio del pensiero greco (amato fin dal liceo). Interesse che emerge limpidamente, ad esempio, nell'ampia reinterpretazione dell'Edipo $R e$, o nella attenta lettura di Artemidoro. Tra le altre, una testimonianza autografa di tale predilezione emerge da una lettera scritta a Fliess il 30 gennaio del 1899 (Freud, 18871904): «Per svago personale leggo la Storia della civiltà greca di Burckhardt, che mi offre paralleli inaspettati. Rimane sempre viva la mia predilezione per l'elemento preistorico in tutte le forme che riguardano l'uomo» (p. 380); e dopo un settimana (il 6 febbraio), in una seconda missiva annotava: «Sono immerso nella Storia della civiltà greca di Burckhardt» (p. 381). Lettura intensa che non solo testimonia limpidamente quanto Freud continuasse a essere

velock Ellis, uno studioso che si interessa dell'argomento del sesso; è evidentemente un uomo molto intelligente, e il cui saggio apparso nell'Alienist and Neurologist (ottobre 1898), che tratta dei rapporti tra isteria e vita sessuale, comincia con Platone e finisce con Freud, dà molta ragione a quest'ultimo e fa degli apprezzamenti molto assennati sugli Studi sull'isteria e sulle successive pubblicazioni» (Freud, 1887-1904, p. 377). 
interessato al pensiero greco, ma che lo condusse ancora una volta a Platone e a La Repubblica: seppur non in relazione al brano di apertura del libro XI, l'opera è richiamata ripetutamente da Burckhardt (1898, volumi 1 e 2), così come il suo autore.

Riepilogando, vi sono molteplici indizi che credo rendano implausibile l'ipotesi di un sostanziale disinteresse nei confronti del brano sul sogno del libro IX de La Repubblica: l'interesse generale per il pensiero greco, che traspare limpidamente dalla lettura di Burckhardt, così come l'ampia reinterpretazione dell'Edipo Re, il cui sogno incestuoso era stato peraltro accostato direttamente da Radestock al passo del libro IX; la consapevolezza che Platone avesse esplicitato il legame tra isteria e sessualità; infine, e direi soprattutto, il fatto che la teoria della reminiscenza avesse rappresentato una fonte di ispirazione per Freud ben prima di iniziare L'interpretazione dei sogni. Indizi che rendono a mio avviso più che plausibile l'ipotesi per cui Freud, incontrata la concezione del sogno del libro IX attraverso Radestock, Büchsenschütz e Cicerone, e posta la pregressa confidenza con La Repubblica, vi abbia dedicato sufficiente attenzione perlomeno per coglierne pienamente la portata. Coltala, avrebbe quindi omesso di riconoscere la genealogia platonica della via regia.

Ma se è così, si può anche ipotizzare che la concezione platonica del sogno quale emersione di desideri repressi, nonché delle sue correlazioni con la follia - ancor più della teoria della reminiscenza e di quella aristotelica della catarsi - abbia potuto rappresentare una fonte di ispirazione tacita per la composizione de L'interpretazione dei sogni: non sappiamo infatti il momento preciso in cui Freud incontrò il libro IX. Al riguardo, va sottolineato che egli potrebbe esser stato stimolato a interessarsi alla questione del sogno nell'antichità molto presto, anche attraverso il legame con Gomperz, avviato a partire dalla traduzione di Mill e poi stretto nei due decenni seguenti (a partire dalla metà degli anni 1880 tenne anche in cura la moglie di Gomperz, Elise, per circa otto anni; vedi Vogel, 1986; Scheidhauer, 1995, pp. 159 sg.). L'antichista viennese si era difatti espressamente occupato del tema fin dal 1866, allorché pubblicò la breve monografia Traumdeutung und Zauberei ("Interpretazione dei sogni e magia"), dedicata soprattutto ad Artedimoro (Gomperz, 1866). Fu poi un allievo del filologo viennese Schenkl e dello stesso Gomperz, Friedrich Salomon Krauss, a curare e tradurre una nuova importante versione del Libro dei sogni di Artemidoro, edita nel 1881; traduzione che Gomperz recensì immediatamente (1881), e che Freud poi citò fin dalla prima edizione de L'interpretazione dei sogni. In breve, sebbene Gomperz e la sua cerchia non misero in evidenza la concezione platonica, rilanciarono il tema del sogno nell'antichità. E così, stimolato a leggere il De Divinatione di Cicerone, o la dissertazione di Radestock, o la panoramica di Büchsenschütz, 
Freud potrebbe aver incontrato il libro IX de La Repubblica in una fase molto precoce della stesura de L'interpretazione dei sogni, o persino prima del suo inizio. Incuriosito o colpito dall'originale concezione di un autore che gli era già stato di ispirazione, nonché agevolato dalla confidenza di lunga data con il grande dialogo, avrebbe potuto facilmente approfondirne la lettura di prima persona, e scoprirvi così le due intuizioni che ritroviamo alla base dell'edificio costruito ne L'interpretazione dei sogni.

\section{Il segreto del folletto}

«In ogni modo ho attuato un proposito che una serie di persone non ancora nate, ma destinate a una cattiva sorte, sentiranno come una grave perdita. Poiché non indovinerai quali persone intendo, te lo dico subito: sono i miei biografi. Ho distrutto tutti i miei appunti e le lettere da quattordici anni a questa parte: gli appunti scientifici e i manoscritti del mio lavoro. (...) I biografi dovranno arrabattarsi, né noi vogliamo rendere loro le cose facili. Ciascuno avrà ragione con le sue idee sull'"evoluzione dell'eroe”, mi diverto già a pensare a come si smarriranno» (Freud, 1837-1939, p. 120).

In questa lettera del 1885 indirizzata a Martha Bernays, divenuta per ironia della sorte ben nota agli storici della psicoanalisi, emerge limpidamente sia la grande ambizione del giovane Freud, sia la sua spiccata ritrosia a svelare le proprie fonti. Ambizione che lo condusse poi a insistere sul primato assoluto della "scoperta" della via regia, marginalizzando radicalmente i contributi precedenti, come si evince da una lettera del 16 maggio 1897 indirizzata a Fliess:

«Ho dato un'occhiata alla letteratura sull'argomento, e mi sono sentito come il folletto celtico: "Ah come sono contento che nessuno, nessuno lo sappia!...”. Nessuno ha avuto il più lieve sospetto che i sogni non siano cose senza senso, bensì appagamenti di desideri» (Freud, 1887-1904, p. 275).

La gioia del folletto freudiano è la gioia del primato. E qui si vede bene anche con quale radicalità Freud volle sminuire fin da subito i precedenti contributi sul tema: erano difatti svariati gli autori che avevano invero già messo ben in luce il fatto che alcuni sogni, sebbene non tutti, non fossero affatto «cose senza senso», ma espressione di particolari desideri (vedi per esempio Goldmann, 2003, cap. IV). La tensione, la difficoltà e l'ambivalenza di Freud nei confronti della letteratura sul sogno emerge del resto in diverse altre lettere indirizzate a Fliess nel periodo di composizione de L'interpretazione dei sogni; in una missiva datata 5 dicembre 1898 leggiamo ad esempio: 
«La letteratura (sul sogno) che sto attualmente leggendo mi rende completamente istupidito. È una punizione terribile, imposta a chiunque voglia scrivere, perché durante la lettura svanisce qualsiasi idea personale. Spesso non riesco a ricordare cosa io abbia scoperto di nuovo; eppure è tutto nuovo in questo campo. Il lavoro di lettura sta assumendo dimensioni incalcolabili» (Freud, 1887-1904, p. 374).

Confusione a cui due giorni dopo (7 dicembre) subentra un giudizio decisamente più drastico e unilaterale: «Del mio umore miserabile (...) sono colpevoli due altri motivi, e cioè la monotonia della pesante attività professionale e la terrificante aridità della letteratura sul sogno che mi resta ancora da leggere» (ibid., p. 375). E ancora, il 28 maggio del 1899, Freud rivendicava senza mezzi termini la paternità della sua "scoperta", marginalizzando o meglio azzerando la portata dell'intera letteratura sul sogno:

«(...) non sono abbastanza ricco da poter tenere per me la migliore - e probabilmente anche l'unica che sia duratura - scoperta che ho fatto. (...) Purtroppo gli dèi, per spaventarmi prima che incominciassi a scrivere il saggio, mi hanno messo davanti la letteratura sul tema. La prima volta mi ci sono arenato, ma questa volta mi farò largo per uscirne; comunque sia, non vi trovo nulla di giusto. Nessuno dei miei lavori è stato mio in modo così completo come questo; mio è il letto del concime, mia la talea e una nova species mihi» (ibid. p. 390).

Posta questa volontà di mantenere il primato assoluto di quella che presentava letteralmente come una "scoperta", credo si possa comprendere perché Freud avrebbe voluto omettere, deliberatamente o meno, di riconoscere l'antica genealogia della via regia. Ma se è così, perché allora citare il «detto di Platone»?

Penso che la fugace inserzione del detto nella penultima pagina dell'opera possa essere interpretata da diverse prospettive; quella per cui propendo è che la citazione rappresenti l'esito di una strategia volta a neutralizzare preventivamente la portata del libro IX. In altri termini, la mia ipotesi è che, una volta colta e assimilata l'analisi platonica, Freud l'abbia confinata, non so se deliberatamente o meno, entro i margini di un detto edificante, depotenziandola. Di certo Freud sapeva perfettamente che nella letteratura scientifica ottocentesca di riferimento sul sogno il testo di Radestock era l'unico, di caratura internazionale e grande diffusione, nel quale veniva richiamato e parzialmente citato il passo saliente del libro IX, peraltro soltanto in nota. Viceversa, nella letteratura principale si privilegiava solitamente un taglio fisiologico (questa è la ragione dei tanti rimandi ad Aristotele), sì che la concezione platonica, incentrata sul ruolo dei desideri repressi di natura soprattutto erotica, risultava anomala e, considerato l'esplicito richiamo al sogno incestuoso, persino scabrosa. In breve, Freud poteva contare sul fatto che il libro IX era una fonte largamente 
trascurata, e invero totalmente ignorata dagli specialisti, e che, nei rarissimi casi in cui vi si faceva riferimento, ci si limitava solitamente a sottolinearne il taglio moralistico ed edificante, come faceva del resto lo stesso Radestock nel corpo del testo, o Büchsenschütz nella sua sintesi, o Cicerone nel suo commento alla lunga citazione (nonché Pfaff). Linea interpretativa che rilanciò con apparente noncuranza.

Questa strategia di neutralizzazione ebbe l'effetto di depistare la critica. Se difatti la concezione platonica era sostanzialmente riconducibile a un detto edificante, non meritava soffermarvisi. Strategia che si è poi rivelata straordinariamente efficace: di fatto, per lungo tempo nessuno storico della psicoanalisi si è dedicato ad analizzare le stringenti analogie tra la «via regia» e la concezione platonica, né tantomeno si è posto la questione di una sua possibile influenza su Freud. Il folletto freudiano, a differenza di quello dei fratelli Grimm che mentre cantava a voce alta svelò il suo segreto (la citazione richiama la favola di Tremotino), continuò a gioire a lungo: nessuno ebbe il minimo sospetto che due delle intuizioni più originali poste alla base de L'interpretazione dei sogni erano state limpidamente delineate da Platone ne La Repubblica. Nel contempo, la citazione del detto platonico potrebbe essere interpretata come una forma di più o meno deliberato autosmascheramento.

Credo altresì che anche l'episodio dell'imperatore romano e del sogno di lesa maestà associato al detto di Platone rientri in questa strategia di depistaggio, deliberato o meno. Freud lo aveva già riportato nel primo capitolo, dedicato alla letteratura scientifica sul sogno, nella sezione dedicata ai sentimenti morali, attraverso il seguente brano tratto dal testo di Friedrich Scholz (1887, p. 36) Schlaf und Traum (“Sonno e sogni”):

«Scholz: "Il sogno è verità: nonostante ogni nobile o vile travestimento riconosciamo sempre il nostro io... L'uomo onesto neppure in sogno può commettere un delitto disonorante o, se giunge a tanto, ne rimane inorridito come di cosa estranea alla sua natura. L'imperatore romano che fece condannare a morte un suo suddito perché questi aveva sognato di decapitare l'imperatore non aveva poi tutti i torti, quando giustificò la condanna dicendo che chi faceva sogni di quel genere certamente aveva pensieri simili durante il giorno. Di qualche cosa che non può trovar posto nel nostro animo, diciamo, ed è significativo: “Ma nemmeno per sogno!”...» (Freud, 1899 p. 71, p. 46 prima ediz. tedesca).

Episodio riportato anche nella dissertazione di Radestock - da cui più che verosimilmente lo riprese lo stesso $\mathrm{Scholz}^{14}$ - e interpretato alla luce della concezione etica di Platone per cui «una persona virtuosa non sognerebbe mai

\footnotetext{
${ }^{14}$ Seppur non nel caso dell'episodio dell'imperatore, Scholz cita Radestock sia nella pagina prima sia in quella dopo averlo narrato (la 36), nonché in diverse altre occasioni (vedi Scholz 1887, pp. 28, 30, 35, 37, 46, 48, 50).
} 
qualcosa di impuro» (Radestock, 1879, p. 163); e poi ripreso anche da Max Leidesdorf (1880), che lo narrava poco dopo aver citato l'«antico detto popolare» per cui «l'uomo buono si permette soltanto in sogno, ciò che il malvagio fa nella veglia» (p. 13).

Ora, questo episodio si sovrappone però perfettamente a quello narrato da Plutarco nella Vita di Dione, in cui il tiranno siracusano Dionisio il Vecchio fece uccidere il suo comandante Marsia perché questi aveva sognato di sgozzarlo, sostenendo che, se lo aveva sognato, doveva averlo anche tramato ${ }^{15}$. Sogno molto originale nel panorama antico ${ }^{16}$, e di taglio chiaramente "platonico": Dionisio interpreta il sogno quale testimonianza dell'emersione di un pensiero che alberga nell'animo di Marsia. L'episodio è del resto narrato sulla scia dell'analisi del libro IX de La Repubblica, usata da Plutarco come modello per descrivere i due tiranni siracusani (Dionisio il Vecchio e il figlio Dionisio il Giovane), ed è associato esplicitamente alla presenza di Platone a Siracusa.

$\mathrm{Al}$ riguardo, la questione per noi più rilevante è data dal fatto che nel periodo in cui componeva L'interpretazione dei sogni Freud molto probabilmente aveva letto la versione originale di Plutarco se non altro attraverso il testo di Albert Lemoine, Du sommeil au point du vue physiologique et psychologique (citato e utilizzato fin dalla prima edizione), che lo riporta fedelmente in un contesto assai significativo del suo lavoro concernente la questione della eticità e della responsabilità morale dei sogni:

«Ma chi sarà colui che vorrà imporre al sognatore questa morale draconiana, e punirlo per dei crimini presunti di cui si sarebbe reso colpevole in sogno, a meno di ragionare come Dionisio il Tiranno, che fece uccidere, dice Plutarco, uno dei suoi capitani, di nome Marsia, "in quanto egli aveva sognato che lo ammazzava, dicendo che questa visione era venuta a lui la notte, mentre dormiva, a causa del fatto che di giorno, nella veglia, l'aveva pensato e si era proposto di farlo" [nota: Plutarco, Vita di Dione, cap. XII]. Accettiamo questo sofisma crudele. Non è dunque affatto per aver sognato di aver ucciso il tiranno che Marsia meritava la morte, ma per averlo voluto uccidere durante la veglia» (Lemoine, 1855, p. 236).

Freud inoltre doveva aver letto anche la storia dei viaggi di Platone presso i tiranni di Siracusa, raccontata dettagliatamente da Burckhardt (1898) nella

\footnotetext{
${ }^{15}$ Scrive Plutarco: «Ed uccise un personaggio che egli stesso aveva elevato a una posizione di comando, Marsia, poiché aveva sognato di ucciderlo: una tale visione, disse, non gli si sarebbe presentata di notte, nel sonno, se durante il giorno non vi avesse pensato e non si fosse proposto di farlo. Proprio così: colui che si adirò con Platone perché non lo definì l'uomo più ardito, aveva poi l'anima timorosa e piena, per viltà, di malizie così grandi» (Vita di Dione, vol. II, p. 11, 9, 3, 961f-962a).

${ }^{16}$ Sull'originalità del sogno, in cui «il sognatore stesso uccide un'altra persona», e sul fatto che Plutarco sia il primo a narrarlo, vedi Gregor Weber (2000, pp. 317-318).
} 
Storia della civiltà greca (vol. 1, pp. 198-213), seppur non in relazione al sogno di Marsia, sì che aveva anche una certa familiarità con il contesto complessivo della questione. Ciò nonostante, egli riportò l'episodio indirettamente, attraverso la citazione letterale del testo di Scholz (che lo riprendeva peraltro in modo estemporaneo), per poi associarlo, nell'ultimo capitolo (sul quale lavorò fino all'ultimo), al «detto di Platone», seguendo così (tacitamente) le due suggestioni di Radestock e di Leidesdorf. In breve, si può sospettare che limitandosi a riprendere e ricombinare gli spunti dei tre interpreti, Freud abbia voluto depistare la critica: inserì sì l'episodio, per lui certamente significativo, ma indirettamente e nella sua versione indeterminata; e inserì anche l'associazione divenuta per così dire canonica con la concezione di Platone, di cui però neutralizzò sincronicamente la portata. Atteggiamento che poi rinverdì nella quarta edizione de L'interpretazione dei sogni, del 1914, allorché subito dopo la citazione di Scholz reinserì seccamente «il detto di Platone»; così come fece nel 1916, nella nona lezione dell'Introduzione alla psicoanali$s i^{17}$. Richiami che risultano ancor più sospetti allorché si consideri che nel 1915, nell'Internationale Zeitschrift für Ärztliche Psychoanalyse, uscì un articolo a firma di Max Nachmansohn interamente dedicato alle analogie tra le concezioni dell'Eros di Platone e Freud, e soprattutto qualora ci si soffermi sul fatto che nel corso degli anni quest'ultimo continuò ad attingere in modo significativo dal lascito platonico, omettendo sistematicamente di riconoscere i debiti via via contratti.

\section{La miniera platonica: omissioni reiterate}

Negli anni 1920 Freud dichiarò a più riprese la decisa convergenza tra il concetto di libido e quello platonico di Eros: nella Prefazione del 1920 alla quarta edizione dei Tre saggi sulla teoria sessuale (Freud, 1905, p. 450), nella lunga e dettagliata discussione del Simposio in Al di là del principio di piacere (Freud, 1920, pp. 242 sg.), incentrata sulla tesi per cui Eros conserva ogni essere vivente, e poi in Psicologia delle masse e analisi dell'Io (Freud, 1921), ove leggiamo che «L' "Eros" del filosofo Platone mostra per la sua provenien$\mathrm{za}$, la sua funzione e il suo rapporto con l'amore sessuale una coincidenza perfetta con la forza amorosa, con la libido della psicoanalisi, come hanno illu-

\footnotetext{
17 «Per Platone invece, gli uomini migliori sono quelli che si limitano a pensare in sogno ciò che gli altri fanno da svegli» (Freud, 1899, p. 71); «O forse non sapete che tutte le soverchierie e le trasgressioni di cui sogniamo durante la notte vengono compiute davvero quotidianamente da uomini svegli in forma di delitti? Che altro fa qui la psicoanalisi se non confermare l'antico detto di Platone secondo il quale i buoni sono coloro che si accontentano di sognare ciò che gli altri, i cattivi, fanno realmente?» (Freud, 1915-17, p. 318).
} 
strato dettagliatamente Nachmansohn e Pfister» (p. 281). Come qui esplicitato, le affinità tra la teoria freudiana e quella platonica erano difatti state messe in luce fin dal 1915 da Nachmansohn in un saggio incentrato appunto sul Simposio. Scoperte le carte, Freud rilanciò, utilizzando Platone anche quale scudo per difendersi dalle sempre più pressanti accuse di pansessualismo, mentre, sincronicamente, lasciava cadere nel vuoto i pur brevi richiami di Nachmansohn (1915, p. 82) all'Eros de La Repubblica e alla biga alata del Fedro. Così come lasciò cadere l'accenno all'inconscio in Platone sottolineato da Oskar Pfister in una lettera inviatagli il 14 gennaio $1921^{18}$, il medesimo anno in cui Pfister rimarcò le coincidenze tra Eros e libido nel suo breve articolo (1921, pp. 264-269).

Ora, tralasciando l'analisi della portata effettiva delle convergenze tra le due visioni dell'Eros, la questione che qui mi preme di più sottolineare è di natura storiografica, e concerne il fatto che le analogie tra le due teorie poggiano in verità su un utilizzo pregresso e tacito del lascito platonico. Freud (1910) era difatti approdato alla tesi per cui l'Eros «conserva ogni cosa vivente» (p. 219) fin dal 1910, come scriveva in Un ricordo d'infanzia di Leonardo da Vinci, sebbene non citasse il Simposio. Pochi mesi prima dell'inizio del saggio su Leonardo, però (cominciato all'incirca nell'ottobre del 1909 e terminato nell'aprile del 1910), in Osservazioni su un caso di nevrosi ossessiva (caso clinico dell'uomo dei topi) (finito nel luglio 1909 ed edito nello stesso anno), analizzando «il rapporto tra il fattore negativo dell'amore e la componente sadica della libido», in modo del tutto inaspettato Freud citava in nota il Simposio:

«Dice Alcibiade di Socrate, nel Simposio: "Quante volte ho desiderato di non vederlo più tra i vivi! Eppure se ciò accadesse, lo so, ne sarei ancora più infelice... tanto inerme, tanto assolutamente inerme, sono di fronte a lui”» (Freud, 1909, p. 69, nota 1).

Credo sia pacifico sostenere che la prima formulazione dell'Eros quale «forza che conserva ogni cosa vivente» sia stata suggerita a Freud dalla lettura del Simposio fatta pochi mesi prima. È assai significativo inoltre il fatto che già nei Tre saggi sulla teoria sessuale (1905, p. 452) egli aveva utilizzato tacitamente il mito aristofaneo (sempre esposto nel Simposio), rimandando in modo vago al «mito poetico della divisione dell'uomo in due metà - uomo e donna - che aspirano a ricongiungersi nell'amore», anziché citare la fonte originaria. Atteggiamento che mostra ancora una volta la ritrosia di Freud a sve-

\footnotetext{
${ }^{18}$ Vedi in particolare la lettera di Pfister a Freud del 14 gennaio 1921, che sottolineava: «Ho fatto in Platone una bella scoperta, che Le farà piacere, Nachmansohn nel suo saggio ha trascurato l'essenziale (...). Egli [Platone] fa risalire all'amore tutto ciò che è arte, religione, morale, e conosce splendidamente anche l'inconscio, le tendenze dell'anima che si incrociano...» (Freud, 1909-39, p. 79).
} 
lare le proprie fonti, nella fattispecie platoniche. Posto che l'origine del mito gli era certamente nota perlomeno fin dal lontano 1883, allorché in una lettera del 25 agosto indirizzata all'allora fidanzata Martha Bernays scriveva: «Sono soltanto un mezzo uomo come dice l'antica favola platonica che tu certo conosci, e la mia sezione soffre non appena sto senza far niente» (Freud, 18371939, p. 41). Richiamo che seppur succinto mostra come fin dai primi anni 1880 Freud avesse presente la fecondità dell'eredità platonica anche da questa prospettiva.

Una seconda omissione, mai riconosciuta ma cristallina, concerne l'immagine della biga alata del Fedro, usata da Platone per esporre il rapporto tra la parte razionale, l'auriga, e le due parti che ne La Repubblica sono definite aggressiva o coraggiosa e desiderante, qui rappresentate da due cavalli, l'uno mansueto e l'altro ribelle, che debbono essere correttamente allevati e guidati, sì che l'auriga possa decidere la direzione da prendere. Viceversa, il cavallo focoso può "costringere» l'auriga e l'altro cavallo a dirigersi ove lui desidera, sì che essi infine «vanno avanti trascinati, cedono e acconsentono a fare ciò che viene loro imposto" (Fedro, 254a-b). Si tratta dunque di un'immagine originale e articolata, che rende conto in termini figurati di una concezione nella quale i desideri, e più in generale le forze pulsionali, vengono a rappresentare i motori energetici di una psiche intesa da una prospettiva eminentemente dinamica e conflittuale. Forze fondamentali, dunque, che la parte razionale deve saper gestire, controllare e indirizzare nella maniera corretta per raggiungere gli obiettivi che si prefigge, e nel contempo per non cadere in loro balia. Immagine tanto significativa quanto nota, che Freud conosceva certamente se non altro attraverso il richiamo di Nachmansohn (1915, p. 77 sg.), e ancor prima di Lemoine ${ }^{19}$ e di Maury ${ }^{20}$ in due lavori citati fin dalla prima edizione de L'interpretazione dei sogni. E immagine che riprese tacitamente, riadattandola, a p. 488 de L'Io e l'Es, edito nel 1922, dunque a ridosso dalle analisi approfondite del Simposio presentate nel 1920 e nel 1921, e che poi rilanciò nuovamente dieci anni più tardi, nella Introduzione alla psicoanalisi (nuova serie di lezioni), edita nel 1932, ove spiega:

\footnotetext{
${ }^{19}$ Scrive Lemoine (1855): «En un mot, elle [l'âme] se gouverne elle-même en gouvernant l'organe, elle gouverne l'organe en obéissant à ses lois, imperat obediendo. Qu'il s'en soit pas toujours ainsi dans la veille, cela est certain; il y a des moments où l'intelligence, Noûs, le cocher de Platon, laisse flotter les rênes et s'abandonne aux caprices de son attelage; il y en a d'autres où celui-ci, Epithymia, surtout, s'emporte, ravissant à sa suite son guide impuissant» (p. 120).

${ }^{20}$ Scrive Maury (1865): «L’homme en enfance st dans un état perpétuel de rêvasserie, et le paroles incohérentes qu'il vous répond doivent être l'expression des idées dont il est bercé. Dès que vous provoquez son attention par une demande, il cherche à reprendre les rênes de ce char intellectuel sur lequel Platon place l'âme: mai il ne peut arriver jusqu'à vous, et il se dirige simplement dans le sens où l'entraînant l'idée qui passant devant son esprit» (p. 107).
} 
«Il rapporto dell'Io con l'Es potrebbe essere paragonato a quello del cavaliere con il suo cavallo. Il cavallo dà l'energia per la locomozione, il cavaliere ha il privilegio di determinare la meta, di dirigere il movimento del poderoso animale. Ma tra l'Io e l'Es si verifica troppo spesso il caso, per nulla ideale, che il cavaliere si limiti a guidare il destriero là dove quello ha scelto di andare» (Freud, 1932, p. 188).

Una terza omissione, tacita ma che credo piuttosto evidente, concerne infine il parallelismo, o meglio il puntuale isomorfismo tra psiche individuale e dimensione sociale e politica sviluppato da Freud in tre saggi scritti tra il 1929 e il 1935. Una posizione che ricalca perfettamente quella de La Repubblica, ove Platone sposta l'asse dell'indagine dalla giustizia del singolo individuo a quella scritta in «caratteri più grandi» nell'intera polis, più facili da leggere e dunque da anteporre ai primi (La Repubblica, 368d-369a), stabilendo poi una perfetta corrispondenza tra le tre parti dell'anima e le tre classi della città ( $i$ bid., 441c sg.), sì da politicizzare la prima e sincronicamente psicologizzare la seconda.

Ne Il disagio della civiltà, del 1929, nel quadro dell'analisi della «analogia tra il processo d'incivilimento e il cammino dello sviluppo individuale», tale per cui «anche la comunità sviluppa un Super-io», Freud sottolinea:

«Qui si verifica addirittura questo caso notevole: otteniamo più dimestichezza con i processi psichici in atto e di essi diventiamo più facilmente consapevoli se li vediamo nella massa piuttosto che nel singolo individuo» (Freud, 1929, pp. 626-627).

E ancora, sempre come Platone, nel breve saggio I miei rapporti con Popper-Lynkeus, del 1932 (p. 311), Freud rovescia il rapporto tra psiche e Stato, politicizzando il primo polo:

«La nostra psiche, quel preziosissimo strumento grazie al quale ci affermiamo nella vita, non rappresenta infatti un'unità felicemente conchiusa in sé medesima, ma è piuttosto paragonabile a uno Stato moderno nel quale una massa gaudente e distruttiva dev'essere tenuta a freno da una classe superiore più assennata» (Freud, 1932, p. 311).

Tre anni dopo, Freud chiude il cerchio, esplicitando a chiare lettere l'isomorfismo:

«Mi resi conto con sempre maggior chiarezza che gli eventi della storia, gli influssi reciproci fra natura umana, sviluppo civile e quei sedimenti di avvenimenti preistorici di cui la religione è il massimo rappresentante, altro non sono che il riflesso dei conflitti dinamici fra Io, Es e Super-Io, studiati dalla psicoanalisi nel singolo individuo: sono gli stessi processi ripresi su uno scenario più ampio» (Freud, 1935, p. 139). 
Qui si tratta quindi non di una vaga convergenza relativa alle classiche analogie tra psiche e società tracciate infinite volte nel corso del cammino del pensiero occidentale, ma piuttosto di una pregnante e precisa sovrapposizione all'isomorfismo presentato da La Repubblica: la psiche individuale diviene più facilmente leggibile allorché la si consideri nel più ampio scenario sociopolitico, con il quale presenta delle puntuali analogie di fondo, mentre la dimensione politica stessa diviene sincronicamente intelligibile allorché la si traduca nei termini delle relazioni, e soprattutto dei conflitti tra le diverse parti dell'anima. Sulla base di tale sovrapposizione, credo si possa così sostenere la tesi per cui l'isomorfismo freudiano rappresenti l'ennesima omissione dell'utilizzo dell'eredità platonica, considerato che una sintesi di tale concezione era molto probabilmente nota a Freud perlomeno fin dagli anni universitari per via del già citato lavoro di Brentano, ove leggiamo:

«Ma spesso anche il corso della storia universale come tale, la successione dei fenomeni legati all'agire delle masse, i progressi e i regressi, l'ascesa e il tramonto dei popoli, possono rendere grossi servigi a chi vuole indagare le leggi generali della natura psichica dell'uomo. Spesso le caratteristiche più importanti della vita psichica si evidenziano maggiormente proprio nelle masse, mentre quelle secondarie scompaiono e si neutralizzano. Già Platone, esaminando lo Stato e la società, sperava di trovarvi scritto a grandi lettere ciò che l'anima del singolo reca scritto in piccolo. Egli riteneva che i tre ambiti della vita psichica corrispondessero alle tre classi fondamentali dello Stato - contadini, guerrieri e governanti - (...). Già abbastanza spesso, e non senza ragione, si è detto che lo sviluppo della storia dell'umanità rappresenta in grande ciò che in modo analogo si ripete, in piccolo, nello sviluppo del singolo. Se è vero che l'osservazione dei fenomeni della società umana getta una luce su quelli psichici del singolo, è tanto più vero l'inverso. In generale, appare più naturale il percorso che porta a trarre conclusioni per la comprensione della società e del suo sviluppo a partire dall'individuo, invece di quello inverso che parte dalla società per giungere a conclusioni sulla psicologia individuale» (Brentano, 1874 p. 106, p. 53 ediz. orig.).

In breve, dagli spunti giovanili tratti dalla teoria della reminiscenza alla ripresa della concezione dell'Eros, dalla metafora del cavaliere all'isomorfismo tra psiche individuale e Stato degli anni 1930, il lascito platonico ha rappresentato una vera miniera per la produzione scientifica di Freud, che nel corso del tempo ha continuato ad attingervi sempre tacitamente. A mezzo stampa si limitò, nei soli casi dell'Eros e del legame tra isteria e sessualità, a esplicitare assai tardivamente le convergenze, presentando entrambe come se fossero a posteriori. Atteggiamento che credo confermi, retrospettivamente, il sospetto che anche la concezione platonica del sogno abbia potuto rappresentare una fonte di ispirazione tacita per la composizione de L'interpretazione dei sogni. 


\section{Un fantasma}

Nonostante i ripetuti incontri con il libro IX; nonostante la confidenza di lunga data con La Repubblica; nonostante l'interesse pregresso per la teoria della reminiscenza e per il mito aristofaneo; nonostante il legame con Gomperz e la sua cerchia; nonostante l'ininterrotto studio del pensiero greco antico; nonostante l'episodio di Marsia e Dionisio; nonostante il desiderio di preservare il primato della "scoperta" della via regia; nonostante l'utilizzo tacito del lascito platonico reiterato nel corso degli anni; nonostante tutto questo, si può continuare a sostenere che Freud non solo non abbia approfondito la lettura del grande dialogo, ma che non abbia neanche colto pienamente la portata della concezione del sogno esposta nel primo brano del libro IX e, dunque, non abbia affatto omesso, deliberatamente o meno, di riconoscere l'antica genealogia della via regia. E anche il silenzio tombale con cui accolse la prima maldestra monografia dedicata alle convergenze tra il suo pensiero e Platone, nel $1934^{21}$, nella quale erano riportate per intero le tre pagine più significative del libro IX (Georgiadès, 1934, pp. 167-171), sarebbe da ascrivere a un sostanziale disinteresse. Il medesimo disinteresse con cui avrebbe riportato, distrattamente, l'episodio dell'imperatore romano narrato da Scholz e il «detto di Platone». Seppur incontrato e persino citato, il libro IX non avrebbe perciò influenzato in alcun modo il pensiero freudiano. Un appuntamento mancato.

Sebbene questa ipotesi sia a mio avviso implausibile a cospetto dell'analisi delle fonti, letture e interessi che abbiamo visto, la si può nondimeno sostenere. In tal caso, a differenza della teoria dell'Eros, della metafora del cavaliere e dell'isomorfismo tra psiche e Stato, evidentemente ripresi tacitamente, nonché dei precoci spunti sulla reminiscenza, Freud avrebbe sì ridato vita a una visione sotterranea alla cultura occidentale, ma in modo fondamentalmente autonomo. Proseguendo in questa direzione, e adottando una prospettiva di taglio scientista, si potrebbe allora essere indotti a considerare la concezione platonica come una conferma transepocale, nell'ambito della civiltà occidentale, dell'universalità dei processi inconsci analizzati da Freud.

Viceversa, restando sul nostro piano storiografico, una seconda ipotesi ermeneutica, che alla luce dei materiali analizzati mi pare decisamente più verosimile della prima, è che Freud possa aver precocemente incontrato e assimilato le due intuizioni platoniche su sogno e follia, e averne poi in certo qual modo dimenticato l'origine, facendole e quindi ritenendole proprie. In questo caso lo straordinario edificio psicoanalitico, che rivoluzionò la figura della

\footnotetext{
${ }^{21}$ Il testo di Patrice Georgiadès (1934) è ancora conservato nella biblioteca di Freud (vedi Davies \& Fichtner 2006, n. catalogo 1496); sulle carenze della trattazione di Georgiadès, vedi per esempio la sua fuorviante tematizzazione dell'inconscio (Georgiadès, 1934, pp. 95 sg.)
} 
soggettività occidentale, riposerebbe così su delle fondamenta antichissime, seppur misconosciute e dimenticate. In altri termini, si potrebbe qui pensare a una sorta di criptomnesia analoga a quella che Freud, ormai anziano, non si sentì di escludere in relazione alla concordanza tra il suo dualismo pulsionale e la teoria di Empedocle:

«So benissimo che la teoria dualistica, la quale postula accanto all'Eros che si manifesta nella libido, e come sua partner avente i medesimi diritti, una pulsione di morte distruttiva o aggressiva, ha trovato in generale scarsa risonanza e non si è propriamente imposta neppure tra gli psicoanalisti. Tanto più, dunque, mi sono rallegrato quando, or non è molto, ho ritrovato la nostra teoria presso uno dei grandi pensatori della Grecia antica. Dinanzi a questa conferma rinuncio volentieri al vanto dell'originalità, tanto più che, data la vastità delle mie letture giovanili, non potrò mai avere la certezza che quel che ho ritenuto essere una mia creazione ex novo non sia stato invece l'effetto di una criptomnesia» (Freud, 1937, p. 528).

Posta però la cruciale importanza attribuita da Freud alla "scoperta" della «via regia», tale per cui egli non pare aver mai voluto rinunciare in alcun modo al «vanto dell'originalità», e posta non solo la generica «vastità» delle sue letture giovanili, ma gli incontri reiterati con il libro IX avvenuti anche durante la stesura de L'interpretazione dei sogni, se anche in questo caso si fosse trattato di una forma di criptomnesia, dovette essere una criptomnesia reiterata e assai particolare. Talmente particolare che mi pare infine più plausibile una terza ipotesi ermeneutica: riconosciuta la priorità della "scoperta" in $\mathrm{La} \mathrm{Re}$ pubblica, Freud avrebbe omesso deliberatamente di riconoscere la genealogia platonica della via regia; inoltre, non si può escludere che la concezione platonica abbia potuto rappresentare persino una fonte tacita di ispirazione. Certo una prova assolutamente dirimente di una lettura attenta del libro IX nella sua interezza da parte del giovane Freud non c'è; credo del resto che l'unica prova certa dovrebbe essere di tipo autobiografico. Tuttavia, preso atto dei materiali qui illustrati, ritengo si possa più che legittimamente sospettare del fantasma che aleggia su L'interpretazione dei sogni. Un fantasma generato fondamentalmente dalla tenace volontà di mantenere deliberatamente nelle proprie mani il primato, a dispetto della limpidezza dei brani platonici incontrati in più occasioni. Se così fosse, la fugace apparizione del «detto di Platone» nella penultima pagina della prima edizione a stampa del capolavoro freudiano dovrebbe allora essere considerata non soltanto come l'esito di una strategia di neutralizzazione e di depistaggio, ma come una sfida rivolta al lettore: un invito criptato da decifrare. 
Riassunto. Muovendo dal richiamo al «detto di Platone» inserito nella penultima pagina della prima edizione de L'interpretazione dei sogni di Freud (1899), vengono preliminarmente esposte le convergenze tra la concezione del sogno di Platone esposta ne La Repubblica e le intuizioni poste alla base dell'edificio freudiano. Alla luce delle fonti testuali citate e utilizzate da Freud, e dei suoi interessi, viene poi avanzata l'ipotesi che egli non soltanto abbia omesso di riconoscere la genealogia teoretica platonica della «via regia che porta alla conoscenza dell'inconscio» (p. 282), ma che l'antico dialogo abbia potuto rappresentare una fonte tacita di ispirazione per la composizione de L'interpretazione dei sogni. [PAROLE CHIAVE: Freud, Platone, sogno, inconscio, storiografia]

Abstract. THE REDISCOVERY OF THE VIA REGIA TO THE UNCONSCIOUS. FREUD READER OF PLATO. Starting with the reference to "Plato's dictum" that Freud added in the second last page of the first edition of The Interpretation of Dreams (1900), the author explains the convergences between the conception of dreams expounded by Plato in The Republic and Freud's fundamental insights. The analysis of bibliographic sources used by Freud, and of his interests, allows us to make the hypotheses that Freud not only omitted the acknowledgment of Plato's theoretical genealogy of "the via regia [royal road] to the unconscious", but also the possibility that The Republic constituted a tacit source of inspiration for the composition of The Interpretations of Dreams. [KEY WORDS: Freud, Plato, dream, unconscious, historiography]

\section{Bibliografia}

Artemidoros aus Daldis (II sec. d.C.). Symbolik der Träume. Übersetzt und mit Anmerkungen begleitet von Friedrich S. Krauss. Wien: A. Hartleben's, 1881.

Assoun P.L. (1976). Freud, la philosophie et les philosophes. Paris: PUF.

Brentano B. (1874). Psychologie vom empirischen Standpunkte, in zwei Bänden. Leipzig: Verlag von Duncker \& Humblot; testo ripreso e ampliato nel 1911 in Klassifikation der Psychischen Phänomene (trad. it.: La psicologia dal punto di vista empirico. Bari: Laterza, 1997, 3 volumi).

Breuer J. \& Freud S. (1892-95). Studi sull'isteria. Opere, 1: 162-439. Torino: Boringhieri, 1967.

Büchsenschütz B. (1868). Traum und Traumdeutung im Alterthume. Berlin: S. Calvary \& Comp.

Burckhardt J. (1898). Griechische Kulturgeschichte. A cura di J. Oeri. Berlin: Sperman, 1898, volumi 1 e 2 (trad. it.: Storia della civiltà greca. Volume I: I Greci e il loro mito. La polis. Torino: Bollati Boringhieri, 2010).

Davies J.K. \& Fichtner G., editors (2006). Freud's Library. A Comprehensive Catalogue / Freuds Bibliothek. Vollständiger Katalog. London: The Freud Museum (Tübingen: Diskord).

Ellis H. (1898). Hysteria in Relation to the Sexual Emotions. The Alienist and Neurologist, 19: 599-615.

Freud S. (1871-81 [1989]). Jugendbriefe an Eduard Silberstein, 1871-1881. Frankfurt a.M.: Fischer, 1989 (trad. it.: “Querido amigo...”. Lettere della giovinezza a Eduard Silberstein, 1871-1881. A cura di M. Conci. Torino: Bollati Boringhieri, 1991).

Freud S. (1873-1939). Lettere alla fidanzata e ad altri corrispondenti, 1873-1939. Torino: Bollati Boringhieri, 1990.

Freud S. (1887-1904 [1985]). The Complete Letters of Sigmund Freud to Wilhelm Fliess, 18871904 (Masson J.M., ed. and transl.). Cambridge: Belknap Press of Harvard Univ. Press, 1985 (trad. it.: Lettere a Wilhelm Fliess, 1887-1904. Torino: Bollati Boringhieri, 1986).

Freud S. (1899 [1900]). L'interpretazione dei sogni. Opere, 3. Torino: Boringhieri, 1966 (prima ediz. orig.: Die Traumdeutung. Leipzig und Wien: Franz Deuticke, 1900).

Freud S. (1905). Tre saggi sulla teoria sessuale. Opere, 4: 443-546. Torino: Boringhieri, 1970. 
Freud S. (1909). Osservazioni su un caso di nevrosi ossessiva (Caso clinico dell'Uomo dei topi). Opere, 6: 1-124. Torino: Boringhieri, 1974.

Freud S. (1909-39). Lettere tra Freud e il pastore Pfister, 1909-1939. Torino: Bollati Boringhieri, 1990.

Freud S. (1910). Un ricordo d'infanzia di Leonardo da Vinci. Opere, 6: 213-276. Torino: Boringhieri, 1974.

Freud S. (1915-17 [1916-17]). Introduzione alla psicoanalisi. Opere, 8: 191-611. Torino: Boringhieri, 1976.

Freud S. (1920). Al di là del principio di piacere. Opere, 9: 189-249. Torino: Boringhieri, 1977.

Freud S. (1921). Psicologia delle masse e analisi dell’Io. Opere, 9: 259-330. Torino: Boringhieri, 1977.

Freud S. (1922 [1923]). L’Io e l’Es. Opere, 9: 471-520. Torino: Boringhieri, 1977.

Freud S. (1924 [1925]). Autobiografia. Opere, 10: 71-141. Torino: Boringhieri, 1978.

Freud S. (1929 [1930]). Il disagio della civiltà. Opere, 10: 555-630. Torino: Boringhieri, 1978.

Freud S. (1932). I miei rapporti con Josef Popper-Lynkeus. Opere, 11: 309-314. Torino: Bollati Boringhieri, 1979.

Freud S. (1932 [1933]). Introduzione alla psicoanalisi (nuova serie di lezioni). Opere, 11: 117284. Torino: Boringhieri, 1979.

Freud S. (1935). Autobiografia. Poscritto del 1935. Opere, 10: 138-141. Torino: Bollati Boringhieri, 1978.

Freud S. (1937). Analisi terminabile e interminabile. Opere, 11: 497-535. Torino: Boringhieri, 1979.

Georgiadès P. (1934). Da Freud a Platon. Paris: Fasquelle.

Gill C. (1985). Plato and the Education of Character. Archiv für Geschichte der Philosophie, 67: 1-26.

Goldmann S. (2003). Via Regia zum Unbewussten. Freud und die Traumforschung im 19. Jahrhundert. Gießen: Psychosozial-Verlag.

Gomperz Th. (1866). Traumdeutung und Zauberei. Wien: Gerold.

Gomperz Th. (1881). Rezension zu Artemidoros aus Daldi, Symbolik der Träume. Wien: A. Hartleben's Verlag. Zeitschrift für die österreichischen Gymnasien, 32: 501-513.

Hemecker W.W. (1991). Vor Freud. Philosophiegeschichtliche Voraussetzungen der Psychoanalyse. München: Philosophia Verlag.

Hildebrandt F.W. (1875). Der Traum und seine Verwertung für's leben. Eine psychologische Studie. Leipzig: Edwin Schloemp.

Hirschmüller A. (1978). Physiologie und Psychoanalyse in Leben und Werk Josef Breuers. Tübingen: Hans Huber.

Jaeger W. (1947). Paideia. Berlin: Walter de Gruyter \& Co. (drittes Buch).

Jones E. (1953-57). The Life and Work of Sigmund Freud (3 volumes). New York: Basic Books, 1953 (vol. I), 1955 (vol. II), 1957 (vol. III) (trad. it.: Vita e opere di Freud. Milano: Il Saggiatore, 1962).

Kenny A. (1969). Mental health in Plato’s Republic. In: The Anatomy of the Soul. Oxford: Basil Blackwell, 1973.

Kofman S. (1990). Miroir et mirages oniriques: Platon, précurseur de Freud. In: Séductions. De Sartre à Héraclite. Paris: Galilée.

Lear J. (1998). Open Minded: Working Out the Logic of the Soul. Cambridge, MA: Harvard University Press.

Lemoine A. (1855). Du sommeil au point du vue physiologique et psychologique. Paris: Baillière.

Leidesdorf M. (1880). Das Traumleben. Vortrag gehalten in Wien zu Gunsten des deutschösterr. Lesevereins der Wiener Hochschulen am 17. März 1880. Wien: Verlag der Alma Mater. 
Maury A. (1865). Le Sommeil et les rêves. Paris: Didier et C.

Mill J.S. (1866). Review of "Platon and the other Companions of Socrates", by George Grote. Edinburgh Review, CXXIII, CCLII (April): 297-364.

Mill J.S. (1880). Gesammelte Werke. Zwölfter Band, Über Frauenemanzipation. Plato. Arbeiterfrage. Socialismus, übersetzt von S. Freud [XII volume: Sull'emancipazione femminile; Platone; La questione del lavoro; Socialismo. Tradotto da Sigmund Freud]. Leipzig: Fues.

Nachmansohn M. (1915). Freuds Libidotheorie verglichen mit der Eroslehre Platos. Internationale Zeitschrift für Ärztliche Psychoanalyse, III, 65: 65-83.

Pfaff E.R. (1868). Das Traumleben und seine Deutung nach den Principien der Araber, Persier, Griechen, Inder und Aegyptier. Leipzig: Denicke.

Pfister O. (1921). Plato als Vorläufer der Psychoanalyse. Internationale Zeitschrift für Psychoanalyse, VII, 3: 264-269.

Plass P. (1978). Anxiety, repression, and morality: Plato and Freud. Psychoanalytic Review, 65, 4: 533-556.

Platone (390-360 a.C.). La Repubblica. Trad. di M. Vegetti. Napoli: Bibliopolis, 2005, 7 vol.

Platone (IV sec. a.C.). Fedro. Trad. di G. Reale. Milano: Mondadori (Fondazione Lorenzo Valla), 1998.

Platone (ca. 360 a.C.), Timeo. Trad. di F. Fronterotta. Milano: Rizzoli, 2006.

Plutarco (ca. 100 d.C.). Vita di Dione. In: Vite parallele. Torino: Einaudi, 1958, vol. II.

Price W. (1990). Plato and Freud. In: Gill C., editor, The Person and the Human Mind. Oxford: Clarendon Press.

Price W. (1995). Mental Conflict. London: Routledge.

Radestock P.W. (1879). Schlaf und Traum. Eine physiologisch-psychologische Untersuchung. Leipzig: Breitkopf und Härtel.

Scheidhauer M. (1995). La période fondamentale de la psychanalyse: un cas exemplaire, Elise Gomperz. In: Fédida P. \& Widlöcher D., editors, Actualité des modale freudiens. Langage, image, pensée. Paris: PUF.

Schellenbacher M. (2011). Sigmund Freud und Franz Brentano. e-journal Philosophie der Psychologie, 15 (März): 1-6; Internet: http://www.jp.philo.at/texte/SchellenbacherM1.pdf.

Scholz F. (1887). Schlaf und Traum: eine populär-wissenschaftliche Darstellung. Leipzig: Mayer.

Simon B. (1973). Plato and Freud. The Mind in Conflict and the Mind in Dialogue. Psychoanalytic Quarterly, 42: 91-122.

Simon B. (1978). Mind and Madness in Ancient Greece. Ithaca, NY: Cornell University Press.

Solinas M. (2008). Psiche: Platone e Freud. Firenze: Firenze University Press.

Solinas M. (2012). Via Platonica zum Unbewussten. Platon und Freud. Wien: Turia \& Kant.

Spitta H. (1882). Die Schlaf und Traumzustände. Tübingen: Fues.

Stella M. (1998). Freud e la "Repubblica": l'anima, la società, la gerarchia. In Vegetti M., a cura di, Platone, La Repubblica. Napoli: Bibliopolis, vol. III.

Tourette, Gilles de la (1891). Traité clinique et thérapeutique de l'hystérie. Vol. 1. Préface de J.-M. Charcot. Paris: E. Plon, Nourrit et Cie (Vol. 2: 1895).

Vogel L.Z. (1986). The Case of Elise Gomperz. American Journal of Psychoanalysis, 46, 3 : 230-238.

Vöhler M. \& Linck D., editors (2009). Grenzen der Katharsis in den modernen Künsten. Transformationen des aristotelischen Modells seit Bernays, Nietzsche und Freud. Berlin: De Gruyter.

Weber G. (2000). Kaiser, Träume und Visionen in Prinzipat und Spätantike. Stuttgart: Franz Steiner. 